\title{
An algorithm for the simulation of thermally coupled low speed flow problems
}

\author{
Pavel Ryzhakov* ${ }^{\dagger}$, Riccardo Rossi and Eugenio Oñate \\ International Center for Numerical Methods in Engineering (CIMNE), Technical University of Catalonia, \\ Barcelona, Spain
}

\begin{abstract}
SUMMARY
In this paper, we propose a computational algorithm for the solution of thermally coupled flows in subsonic regime. The formulation is based upon the compressible Navier-Stokes equations, written in nonconservation form. An efficient modular implementation is obtained by solving the energy equation separately and then using the computed temperature as a known value in the momentum-continuity system. If an explicit single-step time integration scheme for the energy equation is used, the decoupling results to be natural.

Integration of the momentum-continuity system is carried out using a semi-explicit method, combining Runge-Kutta and Backward Euler schemes for the momentum and continuity equations, respectively. Implicit treatment of pressure leads to favorable time step estimates even in the low Mach number $(M a \ll 1)$ regimes. The numerical dissipation introduced by the Backward Euler scheme ensures absence of the spurious high frequencies in the numerical solution.

The key point of the method is the assumption of linear variation of the temperature within a time step. Combined with a fractional splitting of the momentum-continuity system, it allows to solve the continuity only once per time step. Omitting the necessity of solving for the pressure at every intermediate step of the Runge-Kutta scheme minimizes the computational cost associated to the implicit step and leads to an efficiency close to that of a purely explicit scheme.

The method is tested using two benchmark examples. Copyright @ 2011 John Wiley \& Sons, Ltd.
\end{abstract}

Received 3 May 2011; Revised 6 July 2011; Accepted 16 July 2011

KEY WORDS: $\quad$ subsonic flows; semi-explicit methods; natural convection; computational fluid dynamics; $8: 1$ cavity

\section{INTRODUCTION}

Motivation Many problems of practical importance deal with thermally coupled flows at low speeds. These range from heating of liquid semiconductors to ignition of domestic objects under fire situations. In such problems, the flow's velocity is low (typically the subsonic range), but the encountered temperature gradients can be very large. These in turn induce density alterations, thus precluding the use of incompressible models (often used for isothermal low-speed flows). When the temperature gradients and therefore density changes are small, the Boussinesq hypothesis is often employed $[1,2]$. This hypothesis is based on assuming the buoyant term of the momentum equation to be temperature dependent, while keeping the density in the rest of the terms in the governing equations constant. Boussinesq solvers require the subsequent solution of the energy and momentum-continuity system. The popularity of the Boussinesq hypothesis is especially indebted to the simplicity of its implementation, a reason why in many situations, the analysts discard the use of compressible models.

\footnotetext{
*Correspondence to: Pavel Ryzhakov, Technical University of Catalonia, CIMNE, Barcelona, Spain.

$\dagger$ E-mail: pryzhakov@cimne.upc.edu

Copyright @ 2011 John Wiley \& Sons, Ltd.
} 
If the temperature gradients are large and the flow expansion/compression is of interest, one is obliged to use more sophisticated models [3,4]. The use of models based on a full set of compressible Navier-Stokes equations is one of such options. Many of the existing compressible solvers belong to this category, but work well in high-speed regimes only. Mostly being fully explicit, they experience difficulties when applied to the low speed regimes because of the presence of acoustic waves. Although representing the true physical phenomenon, acoustic waves are often of no interest for the macroflow simulations at low speeds. In particular, in the low Mach number regime, they propagate with the speed much larger than that of the bulk flow. Thus, if the objective of the analysis is the prediction of the bulk flow behavior, the acoustic waves can be considered as a sort of 'noise'.

One possibility of excluding the acoustic scale consists in using 'low Mach number models' (see e.g. [5] or [6]). From the mathematical point of view, the idea of the low Mach number models consists in rescaling the full set of compressible Navier-Stokes equations in order to filter out, at the level of PDE, the acoustic pressure. This is done by expanding the compressible equations into Taylor's series and neglecting the terms multiplied by high orders of Mach number, which is valid for $M a \ll 1[7,8]$. The resulting PDE is then different from the original Navier-Stokes equations.

A different option for modeling the low speed flows relies on leaving the governing PDEs unchanged, whereas filtering or damping out the acoustics at the discrete level, for example using a dissipative time integrator. In this case, the numerical solution would contain the acoustic frequencies that can be correctly resolved by the method (FEM in our case), while damping out the spurious frequencies.

This option is followed in this work. In order to obtain an efficient implementation, we introduce a number of assumptions that enable splitting the original monolithic system. These splits include decoupling the energy equation and an application of the fractional step-like split to the

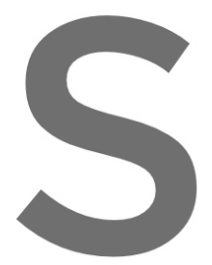
momentum-continuity

The paper is organizet form, written in terns and motivate the de crete form of the go strategy. A fraction step tedhnique is applied
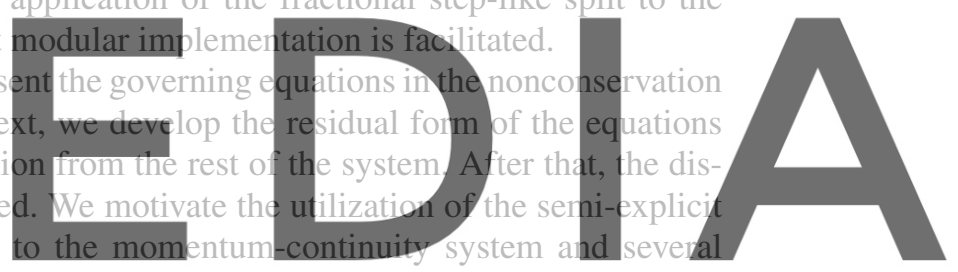

simplifying assumptions are introduced. The paper concludes with two benchmark examples, that Register for free at haldetetholwww.scipedia.com to download the version without the watermark

\section{GOVERNING EQUATIONS IN SUB-SONIC REGIME}

Nonconservation form of the governing equations Because shock waves are not present in subsonic flows, the nonconservation form of the governing equations can be adopted $[9,10]$. The governing equations written in the nonconservation form have the structure of a convectiondiffusion-reaction equation, whose mathematical structure is thoroughly studied and for which the stabilization techniques are well-established. We formulate the governing equations in terms of primitive variables (pressure, velocity, temperature), a typical choice for the low speed formulations [11]. From the implementation point of view, this overall setting enables reusing parts of the incompressible codes that are predominantly written in the nonconservation form and primary variables.

Remark For sonic and super-sonic regime, the use of the conservation form of the governing equations is obligatory because of the presence of the flow discontinuities (shock waves). Note that obtaining the nonconservation form of the governing equations from the conservation, one implies the assumption of differentiability of the variables, which is not valid in case of the presence of discontinuities. It is shown that the solutions of the nonconservation form for $M a \geqslant 1$ do not satisfy the second law of thermodynamics [9], thus explaining why the nonconservation form gives the wrong position and intensity of the shocks, that is, nonphysical results.

The Navier-Stokes equations in the nonconservation form read

$$
\rho \frac{\partial \mathbf{v}}{\partial t}-\nabla \cdot \sigma+\rho(\mathbf{v} \cdot \nabla) \mathbf{v}=\rho \mathbf{f}
$$




$$
\begin{gathered}
\frac{\partial \rho}{\partial t}+\rho \nabla \cdot \mathbf{v}+\mathbf{v} \cdot \nabla \rho=0 \\
\rho c_{v} \frac{\partial T}{\partial t}+\rho(\mathbf{v} \cdot \nabla) T+p \nabla \cdot \mathbf{v}=\nabla \cdot(k \nabla T)+\Psi+Q \\
p=f(\rho, T)
\end{gathered}
$$

where $\rho$ is the density, $\mathbf{v}$ is the velocity, $\boldsymbol{\sigma}$ is the Cauchy stress, $\mathbf{f}$ is the body force, $c_{v}$ is the isochoric heat capacity, $k$ is the conductivity coefficient, $p$ is the pressure, $T$ is the temperature, $\Psi$ is the viscous dissipation energy, $Q$ is the internal heat production term, and $\rho \nabla \cdot \mathbf{v}$ is the work due to flow expansion. The first three conservation equations (momentum, continuity, energy) are completed with the state Equation (4) (not specified until now for the sake of generality), necessary to close the system. We restrict ourselves to Newtonian flows, thus defining the stress tensor as

$$
\sigma=-p \mathbb{I}+2 \mu\left(\frac{\nabla \mathbf{v}+\nabla \mathbf{v}^{T}}{2}\right)
$$

where $\mathbf{I}$ is the identity matrix, $\mu$ is the dynamic viscosity, and $\nabla \mathbf{v}$ is the velocity gradient.

Residual form of the governing equations In the following, we develop the residual form of the governing equations at the continuous level. Such approach gives a clear vision of the terms that couple the equations.

The governing equation system consisting of momentum, continuity, energy and state equations
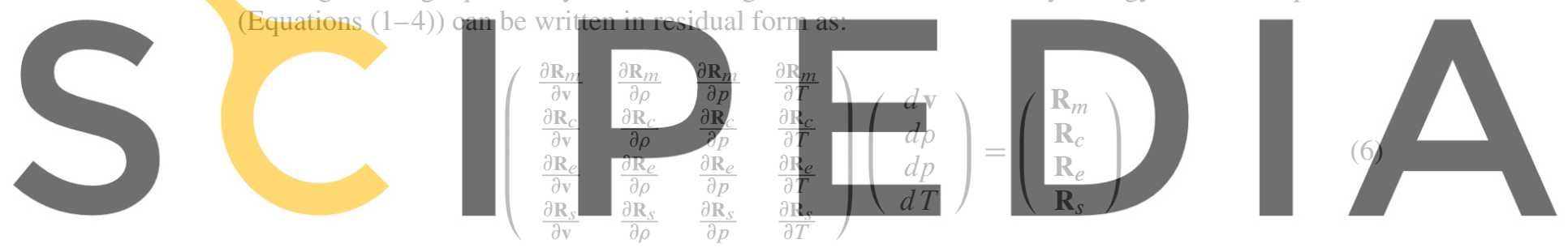

Register for freerath

$$
\begin{gathered}
\mathrm{R}_{m}=\frac{\partial \mathbf{v}}{\partial t}+\mathbf{v} \cdot \nabla \mathbf{v}+\frac{1}{\rho} \nabla p-\frac{1}{\rho} \nabla \cdot(2 \mu \epsilon(\mathrm{v}))-\mathbf{f} \\
\mathbf{R}_{c}=\frac{\partial \rho}{\partial t}+\rho \nabla \cdot \mathbf{v}+\mathbf{v} \cdot \nabla \rho \\
\mathbf{R}_{e}=c_{v} \frac{\partial T}{\partial t}+(\mathbf{v} \cdot \nabla) T+\frac{p}{\rho} \nabla \cdot \mathbf{v}-\frac{1}{\rho} \nabla \cdot(k \nabla T) \\
\mathbf{R}_{s}=p-f(\rho, T)
\end{gathered}
$$

where $\epsilon(\mathbf{v})=\left(\nabla \mathbf{v}+\nabla \mathbf{v}^{T}\right) / 2$. Note that it is convenient to divide the momentum and energy equations by density in order to make the inertial term linear.

Observing Equations (7) and (8), we can notice that $\partial \mathbf{R}_{m} / \partial T=0, \partial \mathbf{R}_{c} / \partial p=0, \partial \mathbf{R}_{c} / \partial T=0$ and $\partial \mathbf{R}_{s} / \partial \mathbf{v}=0$. Thus, the monolithic system simplifies to

$$
\left(\begin{array}{cccc}
\frac{\partial \mathbf{R}_{m}}{\partial \mathbf{v}} & \frac{\partial \mathbf{R}_{m}}{\partial \rho} & \frac{\partial \mathbf{R}_{m}}{\partial p} & 0 \\
\frac{\partial \mathbf{R}_{c}}{\partial \mathbf{v}} & \frac{\partial \mathbf{R}_{c}}{\partial \rho} & 0 & 0 \\
\frac{\partial \mathbf{R}_{e}}{\partial \mathbf{v}} & \frac{\partial \mathbf{R}_{e}}{\partial \rho} & \frac{\partial \mathbf{R}_{e}}{\partial p} & \frac{\partial \mathbf{R}_{e}}{\partial T} \\
0 & \frac{\partial \mathbf{R}_{s}}{\partial \rho} & \frac{\partial \mathbf{R}_{s}}{\partial p} & \frac{\partial \mathbf{R}_{s}}{\partial T}
\end{array}\right)\left(\begin{array}{c}
d \mathbf{v} \\
d \rho \\
d p \\
d T
\end{array}\right)=\left(\begin{array}{c}
\mathbf{R}_{m} \\
\mathbf{R}_{c} \\
\mathbf{R}_{e} \\
\mathbf{R}_{s}
\end{array}\right)
$$


The system is nonlinear and fully coupled (as the off-diagonal terms are nonzero). We can clearly see from Equation (11) that in comparison with the incompressible case, the energy equation becomes fully coupled as it contains the terms that depend on pressure, density, and velocity, thus generally making all the four terms $\left(\partial \mathbf{R}_{e} / \partial \mathbf{v}, \partial \mathbf{R}_{e} / \partial \rho, \partial \mathbf{R}_{e} / \partial p, \partial \mathbf{R}_{e} / \partial T\right)$ in the tangent matrix nonzero.

Up to this point, no approximation was introduced and Equation (11) represents the exact symbolic linearization of the original set of equations.

The implicit solution of the monolithic system defined by Equation (11) is a computationally expensive procedure. The system is large and badly conditioned because of the presence of different variables' scales (pressure, velocity, temperature, density). The fully explicit integration of the monolithic system (typical for sonic flows) is neither favorable because of the stiffness of the mass conservation equation in the low speed regime.

Before defining a feasible time integration algorithm, we propose to obtain a simplified model. Introducing essential approximations, we shall partition the monolithic system. The fundamental idea will be to solve the energy equation separately for the temperature to obtain a reduced system of equations that relates pressure and velocity and finally to use the fractional step approach for the solution of the reduced problem, leading to a computationally efficient modular approach.

Uncoupling the energy equation The first split to the system Equation (6) can be introduced by assuming that the current temperature $T(t+\Delta t)=f(p(t), \rho(t), \mathbf{v}(t))$, that is, treating the temperature explicitly. Introducing the time step index this can be written as

$$
T_{n+1}=f\left(p_{n}, \rho_{n}, \mathbf{v}_{n}\right)
$$
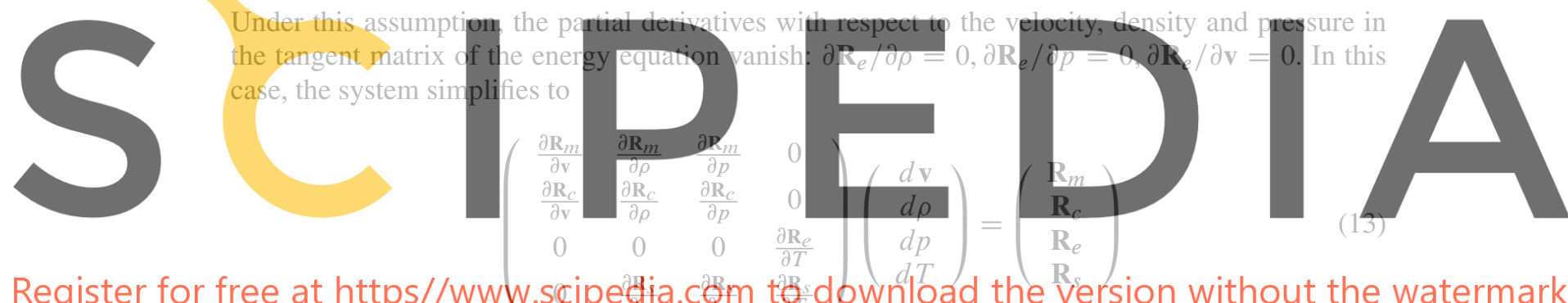

Register for free at https//www.scipegdia.cgm togs download the version without the watermark

Because of the energy equation is now uncoupled, the system of Equations (13) can be written as two separate ones:

$$
\frac{\partial \mathbf{R}_{e}}{\partial T} d T=\mathbf{R}_{e}
$$

and

$$
\left(\begin{array}{ccc}
\frac{\partial \mathbf{R}_{m}}{\partial \mathbf{v}} & \frac{\partial \mathbf{R}_{m}}{\partial \rho} & \frac{\partial \mathbf{R}_{m}}{\partial p} \\
\frac{\partial \mathbf{R}_{c}}{\partial \mathbf{v}} & \frac{\partial \mathbf{R}_{c}}{\partial \rho} & \frac{\partial \mathbf{R}_{c}}{\partial p} \\
0 & \frac{\partial \mathbf{R}_{s}}{\partial \rho} & \frac{\partial \mathbf{R}_{s}}{\partial p}
\end{array}\right)\left(\begin{array}{c}
d \mathbf{v} \\
d \rho \\
d p
\end{array}\right)=\left(\begin{array}{c}
\mathbf{R}_{m} \\
\mathbf{R}_{c} \\
\mathbf{R}_{s}+\left[\frac{\partial \mathbf{R}_{s}}{\partial T} d T\right]_{=0}
\end{array}\right)
$$

Equations (14) and (15) are now coupled only one way via the temperature and can be solved sequentially.

Remark This split is 'exact' if an explicit single step time integration scheme is assumed [12]; however, it can be taken as an approximation $T_{n+1}^{g}$ if an implicit integrator for the temperature is used. In this case, one can use an iterative procedure, where the energy equation solution is repeated after solving momentum/continuity until convergence in density is achieved. In either case, the temperature is used as a known value in the remaining equations system.

Next, we specify a plausible equation of state to enable condensation of the density in order to write the system in terms of exclusively the chosen primary variables $(\mathbf{v}, p, T)$ as unknowns.

In the following, we shall assume the ideal gas equation of state $p=\rho R T$, the most common case 
for the compressible flows [12]. Once the state equation is specified, the Jacobian in Equation (15) can be computed. The residual form of the ideal gas equation can be written as

$$
\mathbf{R}_{s}=p-\rho R T
$$

and thus, the total differential of the density:

$$
d \rho=\frac{1}{R T} d p-\mathbf{R}_{s}
$$

The partial differentiation of the state equation residual Equation (10) with respect to $\rho$ and $p$ gives

$$
\frac{\partial \mathbf{R}_{s}}{\partial p}=1
$$

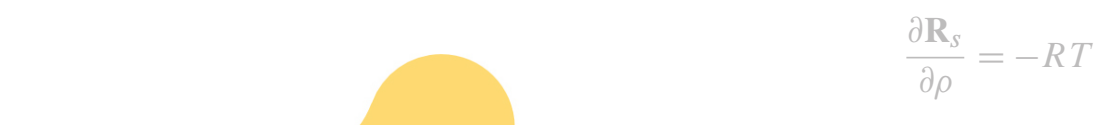

$$
\frac{\partial \mathbf{R}_{s}}{\partial \rho}=-R T
$$

Substituting the partial derivatives of the state equation residual into the system, Equation (15) results in. Next, we substitute Equation (17) into the system. (15), giving
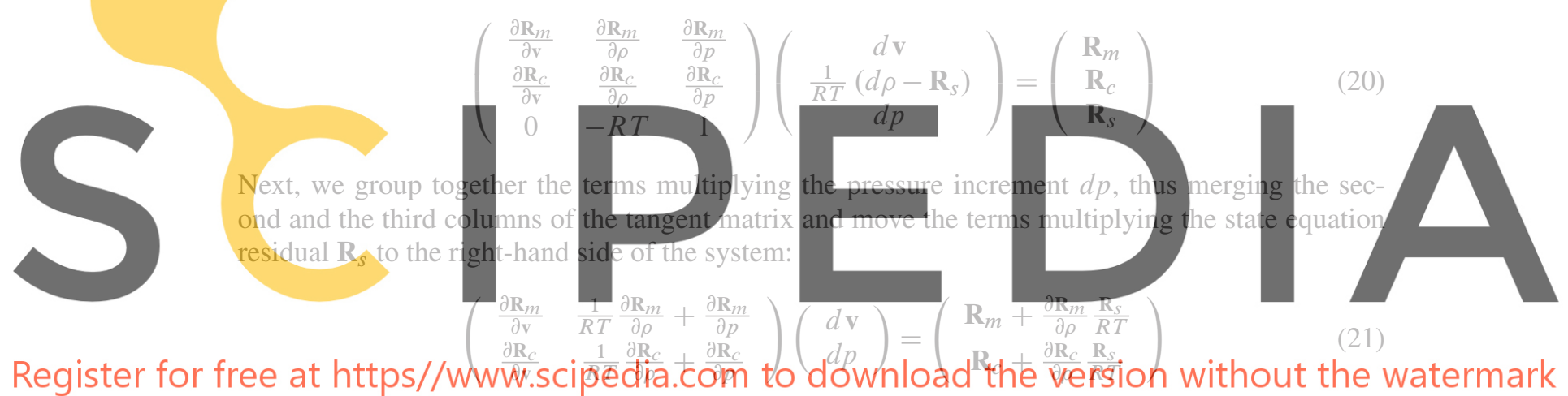

Condensation of the density introduced a term corresponding to the residual of the state equation into the momentum and continuity residua. In a general case, this term might be nonzero.

However, if the temperature at the time instance of the residual evaluation is known and the density in an implicit or a multistep explicit procedure is updated at every intermediate step/iteration via the state equation, this term vanishes. Adopting these assumptions, the system simplifies to

$$
\left(\begin{array}{cc}
\frac{\partial \mathbf{R}_{m}}{\partial \mathbf{v}} & \frac{1}{R T} \frac{\partial \mathbf{R}_{m}}{\partial \rho}+\frac{\partial \mathbf{R}_{m}}{\partial p} \\
\frac{\partial \mathbf{R}_{c}}{\partial \mathbf{v}} & \frac{1}{R T} \frac{\partial \mathbf{R}_{c}}{\partial \rho}+\frac{\partial \mathbf{R}_{c}}{\partial p}
\end{array}\right)\left(\begin{array}{c}
d \mathbf{v} \\
d p
\end{array}\right)=\left(\begin{array}{c}
\mathbf{R}_{m} \\
\mathbf{R}_{c}
\end{array}\right)
$$

Recall that the temperature in the previously mentioned equations is considered a known value because of the assumption in Equation (12).

\section{DISCRETE MODEL}

At this point, the continuous model in the residual form consists of the energy equation one-way coupled to the momentum-continuity system via the temperature. Next, a discrete model will be described. First, we will describe the time integration of the momentum-continuity system. We omit the details of the space discrimination as it is carried out using the standard Galerkin finite element approach. The stabilization, having the energy equation uncoupled, can be carried out separately for the energy and the momentum-continuity equation, using classical stabilization techniques, such as Galerkin/least-squares, various variational multiscale methods Algebraic Subgrid Scale (ASGS), Orthogonal Subscale (OSS) [13,14] or finite calculus (FIC) [15]. 
Semi-explicit approach for the momentum-continuity system The time integration strategy has a major impact upon the computational efficiency of the method based upon Equation (22). A crucial choice consists in whether to derive an explicit or an implicit time integration scheme.

Implicit methods (widely used in the incompressible codes) permit working with large time steps. However, their application to Equation (22) requires evaluation of the partial derivatives with respect to the density in the dynamic tangent, considerably complicating the implementation. Ignoring these partial derivatives, thus violating the consistency of the linearization would impoverish the convergence of the nonlinear procedure.

Explicit approaches require exclusively evaluation of the residua. Thus, the problem associated with the evaluation of the derivatives with respect to the density vanishes. In comparison with the implicit methods, explicit schemes do not involve linear equations system solution, thus making them generally 'faster' per time step than their implicit counterparts. Their limitations originate from the conditional time stability. For sonic and super-sonic flows, explicit schemes are preferred, as the time step estimates (according to the Courant-Friedrich-Levy condition) result to be favorable. For low speed flows, the unfavorable time step restrictions of a fully explicit scheme originate from the presence of the acoustic pressure. The time scale of the acoustic wave for a low speed regime is much smaller than that of the mean flow. Otherwise, the mean flow velocity and viscous scales in the problems of interest permit using large time steps. These considerations lead to a eonclusion that a computationally efficient strategy could be based upon a semi-explicit scheme, where pressure is treated implicitly, whereas the rest of the terms are computed explicitly. Note, that the main computational effort is associated to the solution of the momentum equation, which is a nonlinear vector equation. Obtaining efficiency close to that of a fully explicit scheme implies minimization of the computational cost associated to the implicit steps.
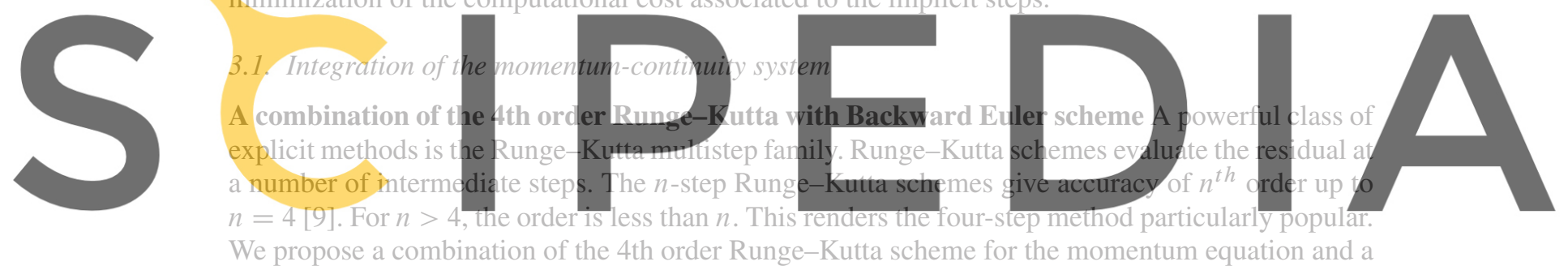

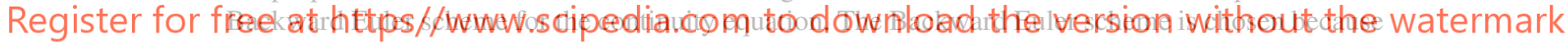
of its dissipative properties. These ensure that the spurious high frequencies (associated with the acoustic scales in a low-speed range) that cannot be resolved by FEM are damped. For a general Cauchy problem of type

$$
\frac{\partial y}{\partial t}=f(y(t), t)
$$

the Runge-Kutta scheme yields the following formula:

$$
\frac{y_{n+1}-y_{n}}{\Delta t}=\frac{1}{6}\left[r_{1}+2 r_{2}+2 r_{3}+r_{4}\right]
$$

where $r_{1} \ldots r_{4}$ are the intermediate residuals (or in other words the 'right-hand side' corrections) that are computed according to the formulae:

$$
\begin{gathered}
r_{1}=f\left(t_{n}, y_{n}\right) \\
y_{\beta 1}=y_{n}+\frac{\Delta t}{2} r_{1} \\
r_{2}=f\left(t_{n}+\frac{\Delta t}{2}, y_{\beta 1}\right) \\
y_{\beta 2}=y_{n}+\frac{\Delta t}{2} r_{2}
\end{gathered}
$$




$$
\begin{gathered}
r_{3}=f\left(t_{n}+\frac{\Delta t}{2}, y_{\beta 2}\right) \\
y_{\beta 3}=y_{n}+\Delta t r_{3} \\
r_{4}=f\left(t_{n}+\Delta t, y_{\beta 3}\right)
\end{gathered}
$$

This means that the computation of $y_{n+1}$ requires evaluating the residual four times and updating the unknown at each of the intermediate steps.

The momentum Equation (1) assumes the form of Equation (23), thus Equation (25) can be applied directly, leading to the following semidiscrete momentum equation residual (note that with a certain abuse of notation, we use the same symbol $\mathbf{R}_{m}$ for the semidiscrete and continuous residual):

$$
\mathbf{R}_{m}=\frac{\mathbf{v}_{n+1}-\mathbf{v}_{n}}{\Delta t}-\frac{1}{6}\left[\mathbf{r}_{1}+2 \mathbf{r}_{2}+2 \mathbf{r}_{3}+\mathbf{r}_{4}\right]=0
$$

The intermediate residua (that will be distinguished by a minuscule r symbol), defined according to Equation (7) are substituted into Equations (25-31) giving:

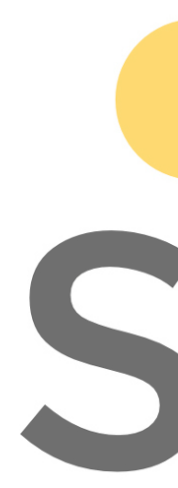

$$
\mathbf{r}_{1}=\mathbf{f}-\mathbf{v}_{n} \cdot \nabla \mathbf{v}_{n}-v \Delta \mathbf{v}_{n}+\frac{1}{\rho_{n}} \nabla p_{n}
$$

$$
\mathbf{v}_{\beta 1}=\mathbf{v}_{n}+\Delta t \mathbf{r}_{1}
$$

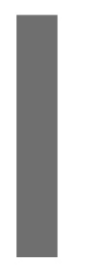

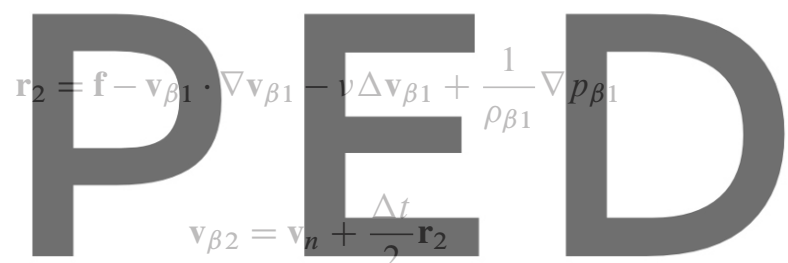

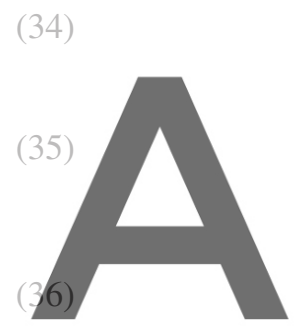

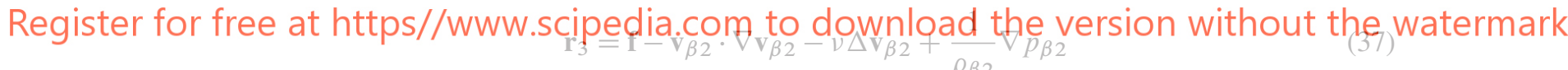

$$
\begin{gathered}
\mathbf{v}_{\beta 3}=\mathbf{v}_{n}+\frac{\Delta t}{2} \mathbf{r}_{3} \\
\mathbf{r}_{4}=\mathbf{f}-\mathbf{v}_{\beta 3} \cdot \nabla \mathbf{v}_{\beta 3}-v \Delta \mathbf{v}_{\beta 3}+\frac{1}{\rho_{n+1}} \nabla p_{n+1}
\end{gathered}
$$

Equations (33-39) contain intermediate pressure and density $p_{\beta i}, \rho_{\beta i}$. Therefore, in principle, the continuity equation must be solved at every intermediate step. This would considerably increase the computational cost, requiring to perform the implicit step (i.e. solve the continuity Equation (69)) three times. Additionally, the computation of the intermediate density via the constitutive relation requires the knowledge of the temperature at $t+\Delta t / 2$.

However, this is unknown as the solution of the decoupled energy equation provides only $T_{n+1}=$ $T(t+\Delta t)$. Thus, we are obliged to introduce an assumption upon the variation of the temperature between $t_{n}$ and $t_{n+1}$. We propose to use a linear approximation $T_{t+(\Delta t / 2)}=\left(T_{n}+T_{n+1}\right) / 2$ (because $\beta_{2}$ and $\beta_{3}$ correspond to $t=t_{n}+(\Delta t / 2)$ ). This in turn implies (according to the state equation) that $p / \rho$ varies linearly in time between $t_{n}$ and $t_{n+1}$, and thus also does the term $1 \rho / \nabla p$. This leads to the following fundamental simplification:

$$
\frac{1}{\rho_{\beta 1}} \nabla p_{\beta 1}=\frac{1}{\rho_{\beta 2}} \nabla p_{\beta 2}=\frac{1}{\rho} \nabla p\left(t_{n}+\frac{\Delta t}{2}\right)=0.5\left(\frac{1}{\rho_{n}} \nabla p_{n}+\frac{1}{\rho_{n+1}} \nabla p_{n+1}\right)
$$


Application of Equation (40) in Equations (33-39) simplifies the intermediate residua to:

$$
\begin{gathered}
\mathbf{r}_{1}=\mathbf{f}-\mathbf{v}_{n} \cdot \nabla \mathbf{v}_{n}-v \Delta \mathbf{v}_{n}+\frac{1}{\rho_{n}} \nabla p_{n} \\
\mathbf{r}_{2}=\mathbf{f}-\mathbf{v}_{\beta 1} \cdot \nabla \mathbf{v}_{\beta 1}-v \Delta \mathbf{v}_{\beta 1}+\frac{1}{2}\left(\frac{\nabla p_{n}}{\rho_{n}}+\frac{\nabla p_{n+1}}{\rho_{n+1}}\right) \\
\mathbf{r}_{3}=\mathbf{f}-\mathbf{v}_{\beta 2} \cdot \nabla \mathbf{v}_{\beta 2}-v \Delta \mathbf{v}_{\beta 2}+\frac{1}{2}\left(\frac{\nabla p_{n}}{\rho_{n}}+\frac{\nabla p_{n+1}}{\rho_{n+1}}\right) \\
\mathbf{r}_{4}=\mathbf{f}-\mathbf{v}_{\beta 3} \cdot \nabla \mathbf{v}_{\beta 3}-v \Delta \mathbf{v}_{\beta 3}+\frac{1}{\rho_{n+1}} \nabla p_{n+1}
\end{gathered}
$$

Equations (41-44) now contain densities and pressures corresponding to exclusively $t_{n}$ and $t_{n+1}$. It is convenient now to take out the pressure gradients (which is of particular advantage in the perspective of applying the fractional split) out of the residua, rewriting thus the Runge-Kutta equations as:

$$
\frac{\mathbf{v}_{n+1}-\mathbf{v}_{n}}{\Delta t}=\frac{1}{6}\left[\mathbf{r}_{1}^{\prime}+2 \mathbf{r}_{2}^{\prime}+2 \mathbf{r}_{3}^{\prime}+\mathbf{r}_{4}^{\prime}\right]+\frac{1}{2}\left(\frac{\nabla p_{n}}{\rho_{n}}+\frac{\nabla p_{n+1}}{\rho_{n+1}}\right)
$$

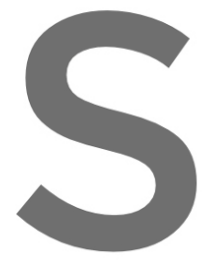

with the intermediate residua $\mathbf{r}_{i}^{\prime}$ (note that the intermediate velocities are defined as before, that is,
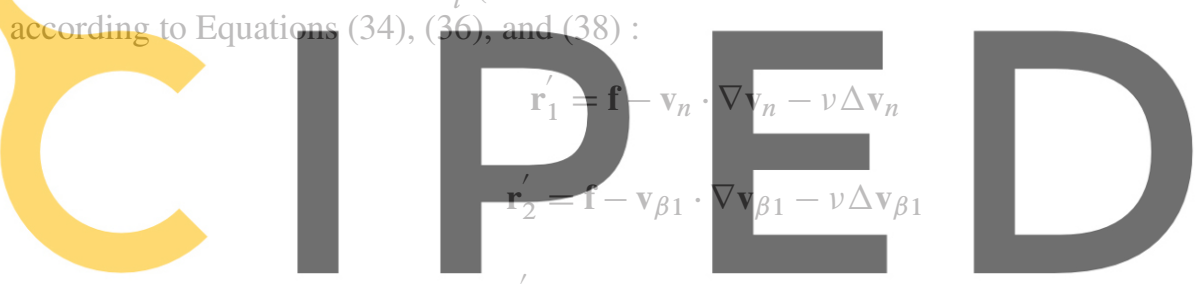

$\mathbf{r}_{3}^{\prime}=\mathbf{f}-\mathbf{v}_{\beta 2} \cdot \nabla \mathbf{v}_{\beta 2}-v \Delta \mathbf{v}_{\beta 2}$
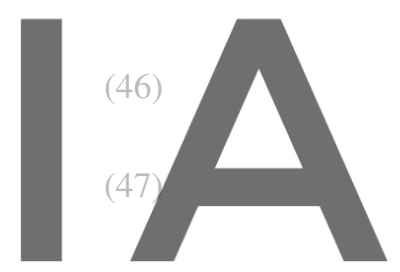

Register for free at https//www.scipedia.com to download the version without the watermark $\mathbf{r}_{4}=\mathbf{f}-\mathbf{v}_{\beta 3} \cdot \nabla \mathbf{v}_{\beta 3}-v \Delta \mathbf{v}_{\beta 3}$

Equations (45) and (46-49) constitute the basis for the Finite Element (FE) formulation that follows.

\subsection{Discrete form}

The discrete form of Equations (45-49) using linear pressure-velocity interpolations reads (note that the discrete quantities are distinguished by an over-bar notation)

$$
\mathbf{M} \frac{\overline{\mathbf{v}}_{n+1}-\overline{\mathbf{v}}_{n}}{\Delta t}-\frac{1}{6}\left[\overline{\mathbf{r}}_{1}+2 \overline{\mathbf{r}}_{2}+2 \overline{\mathbf{r}}_{3}+\overline{\mathbf{r}}_{4}\right]+\frac{1}{2}\left[G_{1 / \rho_{n}} P_{n}+G_{1 / \rho_{n+1}} P_{n+1}\right]
$$

which is computed in four successive steps, evaluating the discrete intermediate residua and velocities defined as

$$
\begin{gathered}
\overline{\mathbf{r}}_{1}=\mathbf{F}-\mathbf{K}\left(\overline{\mathbf{v}}_{n}\right)\left(\overline{\mathbf{v}}_{n}\right)-v \mathbf{L} \overline{\mathbf{v}}_{n} \\
\overline{\mathbf{v}}_{\beta 1}=\overline{\mathbf{v}}_{n}+\mathbf{M}^{-1} \frac{\Delta t}{2}\left[\overline{\mathbf{r}}_{1}+\mathbf{G}_{1 / \rho_{n}} \bar{p}_{n}\right] \\
\overline{\mathbf{r}}_{2}=\mathbf{F}-\mathbf{K}\left(\overline{\mathbf{v}}_{\beta 1}\right)\left(\overline{\mathbf{v}}_{\beta 1}\right)-v \mathbf{L}_{\left(1 / \bar{\rho}_{\beta 1}\right)} \overline{\mathbf{v}}_{\beta 1} \\
\overline{\mathbf{v}}_{\beta 2}=\overline{\mathbf{v}}_{n}+\mathbf{M}^{-1} \frac{\Delta t}{2}\left[\overline{\mathbf{r}}_{2}+\frac{1}{2}\left(G_{1 / \rho_{n}} P_{n}+G_{1 / \rho_{n+1}} P_{n+1}\right)\right]
\end{gathered}
$$




$$
\begin{gathered}
\overline{\mathbf{r}}_{3}=\mathbf{F}-\mathbf{K}\left(\overline{\mathbf{v}}_{\beta 2}\right)\left(\overline{\mathbf{v}}_{\beta 2}\right)-\nu \mathbf{L}_{\left(1 / \bar{\rho}_{\beta 2}\right)} \overline{\mathbf{v}}_{\beta 2} \\
\overline{\mathbf{v}}_{\beta 3}=\overline{\mathbf{v}}_{n}+\mathbf{M}^{-1} \frac{\Delta t}{2}\left[\overline{\mathbf{r}}_{3}+\frac{1}{2}\left(G_{1 / \rho_{n}} P_{n}+G_{1 / \rho_{n+1}} P_{n+1}\right)\right] \\
\overline{\mathbf{r}}_{4}=\mathbf{F}-\mathbf{K}\left(\overline{\mathbf{v}}_{\beta 3}\right)\left(\overline{\mathbf{v}}_{\beta 3}\right)-\nu \mathbf{L} \overline{\mathbf{v}}_{\beta 3}
\end{gathered}
$$

where $\mathbf{M}, \mathbf{L}, \mathbf{G}, \mathbf{K}$ are the global mass, Laplacian, gradient matrices, and discrete convective operator, respectively, and $\mathbf{F}$ is the body force vector, assembled from the elemental contributions defined as (N stands for the standard linear FE shape functions)

$$
\begin{gathered}
\mathbf{M}=\int_{\Omega_{e}} \mathbf{N N}^{T} d \Omega \\
\mathbb{L}=\int_{\Omega_{e}} \nabla \mathbf{N} \nabla \mathbf{N}^{T} d \Omega \\
\mathrm{G}=\int_{\Omega_{e}} \nabla \mathbf{N N} d \Omega
\end{gathered}
$$
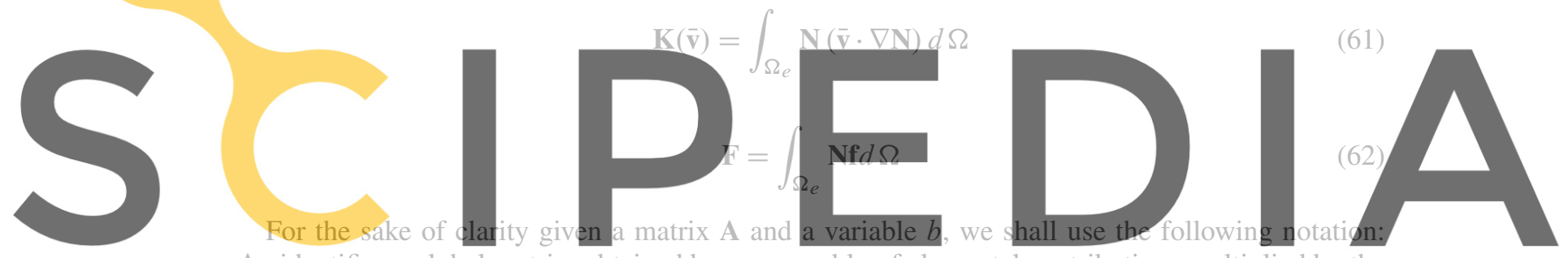

$\mathbf{A}_{b}$ identifies a global matrix, obtained by an assembly of elemental contributions multiplied by the

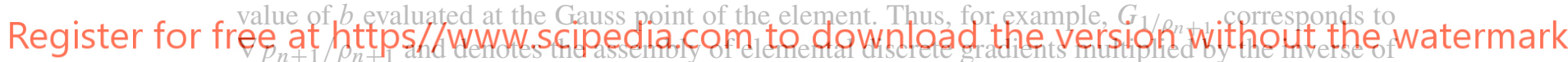

the density $1 / \rho_{n+1}$ taken at the Gauss point, that is, $G_{1 / \rho_{n+1}}=\int_{\Omega} \nabla \mathbf{N N}\left(1 / \rho^{G}\right) d \Omega$.

Equations (50-57) define the discrete model of the momentum equation under the fundamental assumption (40), which permitted us to formulate the intermediate residua without necessitating the solution of the continuity equations at the intermediate steps.

Next, we shall specify the discrete version of the continuity equation.

Continuity equation Application of the Backward Euler scheme to the continuity Equation (8) gives the following residual

$$
\mathbf{R}_{c}=\frac{\rho_{n+1}-\rho_{n}}{\Delta t}+\rho_{n+1} \nabla \cdot \mathbf{v}_{n+1}+\mathbf{v}_{n+1} \cdot \nabla \rho_{n+1}
$$

The discrete version of the residual is

$$
\overline{\mathbf{R}}_{c}=\mathbf{M} \frac{\bar{\rho}_{n+1}-\bar{\rho}_{n}}{\Delta t}+\mathbf{D}_{\rho_{n+1}} \overline{\mathbf{v}}_{n+1}+\mathbf{C}\left(\overline{\mathbf{v}}_{n+1}\right) \bar{\rho}_{n+1}
$$

where $\mathbf{D}$ is the divergence matrix, and $\mathbf{C}$ is the scalar convective operator assembled from the following elemental contributions:

$$
\begin{gathered}
\mathbf{D}=\int_{\Omega_{e}} \mathbf{N} \nabla \mathbf{N} d \Omega \\
\mathbf{C}(\overline{\mathbf{v}})=\int_{\Omega_{e}} N(\overline{\mathbf{v}} \cdot \nabla \mathbf{N}) d \Omega
\end{gathered}
$$


Next, we evaluate the approximate tangent entries according to Equation (22):

$$
\begin{gathered}
\frac{\partial \overline{\mathbf{R}}_{c}}{\partial \overline{\mathbf{v}}} \approx \mathbf{D}_{\left(\rho_{n+1}\right)} \\
\frac{1}{R \bar{T}_{n+1}} \frac{\partial \overline{\mathbf{R}}_{c}}{\partial \bar{\rho}}+\frac{\partial \overline{\mathbf{R}}_{c}}{\partial \bar{p}} \approx \frac{1}{R T_{n+1}}\left[\frac{1}{\Delta t} \mathbf{M}+\mathbf{C}\left(\overline{\mathbf{v}}_{n+1}\right)\right]
\end{gathered}
$$

Now, we introduce the residual (64) and the derivatives (67-68) into Equation (22) (second line of the matrix equation) and write the continuity equation in terms of pressure:

$$
\begin{array}{r}
\mathbf{D}_{\rho_{n+1}} d \overline{\mathbf{v}}+\left[\frac{1}{R \bar{T}_{n+1}}\left(M+\mathbf{C}\left(\overline{\mathbf{v}}_{n+1}\right)\right)\right] d \bar{p}= \\
=\frac{1}{R \bar{T}_{n+1}} M \frac{\bar{p}_{n+1}-\bar{p}_{n}}{\Delta t}+\mathbf{D}_{\rho_{n+1}} \overline{\mathbf{v}}_{n+1}+\frac{1}{R \bar{T}_{n+1}} \mathbf{C}\left(\overline{\mathbf{v}}_{n+1}\right) \bar{p}_{n+1}
\end{array}
$$

Fractional step split The discrete form of the coupled momentum-continuity system Equation (22) is the combination of Equation (69) and (50-57). In order to decouple the solution for the velocity and pressure, we apply the pressure splitting, introducing the fractional velocity $\tilde{\mathbf{v}}$. The fractional and end-of-step momentum equations can be written as:

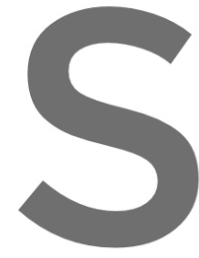

$$
\mathbf{M} \frac{\tilde{\mathbf{v}}-\overline{\mathbf{v}}_{n}}{\Delta t}=\left[\tilde{\mathbf{r}}_{1}+2 \tilde{\mathbf{r}}_{2}+2 \tilde{\mathbf{r}}_{3}+\tilde{\mathbf{r}}_{4}\right]+\mathrm{G}_{1 / \rho_{n}} \bar{p}_{n}
$$
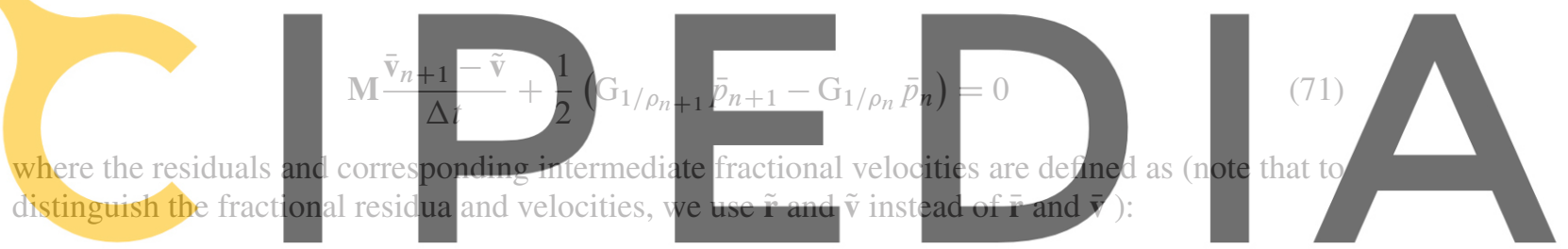

$$
\tilde{\mathbf{r}}_{1}=\mathbb{F}-\mathbb{K}_{\overline{\mathbf{v}}_{n}} \overline{\mathbf{v}}_{n}-\mu \mathbf{L}_{1 / \rho_{n}} \overline{\mathbf{v}}_{n}
$$

Register for free at https//www.scipedia.com to download the version without the watermark

$$
\begin{gathered}
\tilde{\mathbf{v}}_{\beta 1}=\overline{\mathbf{v}}_{n}+\mathrm{M}^{-1} \frac{\Delta t}{2}\left[\tilde{\mathbf{r}}_{1}+\mathrm{G}_{\rho_{n}} \bar{p}_{n}\right] \\
\tilde{\mathbf{r}}_{2}=\mathbf{F}-\mathrm{K}_{\tilde{\mathbf{v}}_{\beta 1}} \tilde{\mathbf{v}}_{\beta 1}-\mu L_{1 / \rho_{n}} \tilde{\mathbf{v}}_{\beta 1} \\
\tilde{\mathbf{v}}_{\beta 2}=\overline{\mathbf{v}}_{n}+\mathbf{M}^{-1} \frac{\Delta t}{2}\left[\tilde{\mathbf{r}}_{2}+0.5\left(G_{\rho_{n}} \bar{p}_{n}\right)\right] \\
\tilde{\mathbf{r}}_{3}=\mathbf{F}-\mathrm{K}_{\tilde{\mathbf{v}}_{\beta 2}} \tilde{\mathbf{v}}_{\beta 2}-\mu \mathrm{L}_{1 / \rho_{n}} \tilde{\mathbf{v}}_{\beta 2} \\
\tilde{\mathbf{v}}_{\beta 3}=\overline{\mathbf{v}}_{n}+\mathbf{M}^{-1} \Delta t\left[\tilde{\mathbf{r}}_{3}+0.5\left(G_{\rho_{n}} \bar{p}_{n}\right)\right] \\
\tilde{\mathbf{r}}_{4}=\mathbf{F}-\mathrm{K}_{\tilde{\mathbf{v}}_{\beta 3}} \tilde{\mathbf{v}}_{\beta 3}-\mu \mathrm{L}_{1 / \rho_{n}} \tilde{\mathbf{v}}_{\beta 3}
\end{gathered}
$$

One can see, that the fractional momentum equation and the intermediate residua contain pressure and density corresponding exclusively to the time step $n$.

To express the end-of-step velocity as a function of the introduced fractional velocity, we use Equation (71):

$$
\overline{\mathbf{v}}_{n+1}=\frac{\Delta t}{2} M^{-1}\left[G_{1 / \rho_{n}} P_{n}-G_{1 / \rho_{n+1}} \bar{p}_{n+1}\right]+\tilde{\mathbf{v}}
$$


Substituting Equation (79) into the continuity Equation (69), we obtain the pressure Poisson equation:

$$
\begin{aligned}
& \mathbf{D}_{\rho_{n+1}}\left[\tilde{\mathbf{v}}-\frac{\Delta t}{2} M^{-1}\left(G_{1 / \rho_{n+1}} \bar{p}_{n+1}-G_{1 / \bar{p}_{n}} \bar{P}_{n}\right)\right]+ \\
& +\frac{1}{R \bar{T}_{n+1} \Delta t} M\left[\bar{p}_{n+1}-\bar{p}_{n}\right]+\frac{1}{R \bar{T}_{n+1}} \mathrm{C}(\tilde{\mathbf{v}}) \bar{p}_{n+1}=0
\end{aligned}
$$

Here, we need to introduce the approximations $\mathbf{G}_{\rho_{n+1}} \approx \mathbf{G}_{\rho_{n}}$ and $\mathbf{D}_{\rho_{n+1}} \approx \mathbf{D}_{\rho_{n}}$, in order to make continuity equation linear in the pressure. As usually, the discrete Laplacian is approximated as $\mathbf{D M}^{-1} \mathbf{G} \approx \mathbf{L}$.

Substituting these in Equations (70-71) leads to the final system of three equations.

$$
\begin{gathered}
\mathbf{M} \frac{\tilde{\mathbf{v}}-\overline{\mathbf{v}}_{n}}{\Delta t}=\frac{1}{6}\left[\tilde{\mathbf{r}}_{1}\left(\overline{\mathbf{v}}_{n}\right)+2 \tilde{\mathbf{r}}_{2}\left(\overline{\mathbf{v}}_{\beta 1}\right)+2 \tilde{\mathbf{r}}_{3}\left(\overline{\mathbf{v}}_{\beta 2}\right)+\tilde{\mathbf{r}}_{4}\left(\overline{\mathbf{v}}_{\beta 3}\right)\right]+G_{1 / \rho_{n}} \bar{p}_{n} \\
\frac{1}{2} \mathbf{L}_{\rho_{n}}\left(\bar{p}_{n+1}-\bar{p}_{n}\right)+\frac{1}{R \bar{T}_{n+1} \Delta t} M \bar{p}_{n+1}+\frac{1}{R \bar{T}_{n+1}} \mathbf{C}_{(\tilde{\mathbf{v}})} \bar{p}_{n+1}= \\
=\frac{1}{R \bar{T}_{n+1} \Delta t} \mathbf{M} \bar{p}_{n}+\mathbf{D}_{\rho_{n}} \tilde{\mathbf{v}} \\
\mathbf{M} \frac{\overline{\mathbf{v}}_{n+1}-\tilde{\mathbf{v}}}{\Delta t}+\frac{1}{2}\left(G_{1 / \rho_{n+1}} \bar{p}_{n+1}-G_{1 / \rho_{n}} \bar{p}_{n}\right)=0
\end{gathered}
$$

\subsection{Energy equation integration and the overall solution strategy}

Decoupling the energy equation necessitated to assume that the derivative of the residual $\mathbf{R}_{e}$ is nonzero only with respect to the temperature (assumption Equation (12)), thus treating all the rest of the terms explicitly. This is automatically satisfied for the simplest explicit scheme, namely the Forward Euler scheme. In this case the discrete version of the energy equation Equation (8) has the following form:

$$
\overline{\mathbf{R}}_{e}=c_{v} \mathbf{M} \frac{\bar{T}_{n+1}-\bar{T}_{n}}{\Delta t}-\left(\mathbf{C}\left(\overline{\mathbf{v}}_{n}\right) \bar{T}_{n}+R \mathrm{D}_{T_{n}} \overline{\mathbf{v}}_{n}-k \mathrm{~L}_{1 / \rho_{n}} \bar{T}_{n}\right)=0
$$

and its solution gives the end-of-step temperature $T_{n+1}$ without introducing any approximation error, that is, the precision is bounded only by the order of the used time scheme.

Although for the Forward Euler scheme the splitting is exact, it restricts the time accuracy in temperature to the first order and introduces time step restrictions because of conditional stability. It may not be convenient to have the time step controlled both by the estimate originating from the momentum equation integration (Runge-Kutta) and the energy equation. These considerations lead to the possible choice of solving the energy equation implicitly, within an iterative context. That is, solving the energy equation given the predicted values of pressure, density, and velocity, obtaining $T_{n+1}^{i}(i=0$, that is the first guess upon the end-of-step temperature). Either second-order predictions can be applied (e.g. $\rho_{n+1}^{0} \approx \alpha \rho_{n}-\beta \rho_{n-1}$, with $\alpha=2, \beta=1$ for constant time step size) or simply the value at $n$ can be used $\left(\rho_{n+1} \approx \rho_{n}\right)$. Given $T_{n+1}^{0}$, the momentum-continuity system is solved to give $\mathbf{v}_{n+1}^{i}, p_{n+1}^{i}$ and density $\rho_{n+1}^{i}=\frac{p_{n+1}^{i}}{R T_{n+1}^{i}}$ is recovered. Then, the energy equation is solved using these updated values, and the whole procedure is repeated until convergence in terms of density is achieved.

The algorithms based on both possibilities for uncoupling the energy equation are presented in Tables I and II.

Solution algorithms An algorithm based on the explicit one-step integration of the energy equation is presented in Table I.

For the solution of the examples in the next section, single-step algorithm was used. 
Table I. Implementation of the semi-explicit solver using explicit (Forward Euler) integration of the energy equation.

1. Solve the energy equation explicitly (Equation (84)) and obtain the temperature $\bar{T}_{n+1}=f\left(\bar{v}_{n}, \bar{\rho}_{n}, \bar{T}_{n}\right)$

2. Solve the fractional momentum equation (Equation (81)), obtain $\tilde{\mathbf{v}}$ (using $\bar{\rho}=\bar{\rho}_{n}$ for all the intermediate steps)

3. Solve the continuity equation for the pressure (Equation (82)), obtain $\bar{p}_{n+1}$

4. Obtain $\bar{\rho}_{n+1}$ from the ideal gas equation (Equation (4))

5. Solve for end-of-step momentum (Equation (83)) and obtain $\overline{\mathbf{v}}_{n+1}$

Table II. Implementation of the semi-explicit solver using implicit integration of the energy equation.

1. Solve the energy equation implicitly using approximations for $\bar{p}_{n+1}^{0}, \bar{\rho}_{n+1}^{0}, \overline{\mathbf{v}}_{n+1}^{0}$ (Equation (84)) and obtain a 'guess' for the end-of-step temperature $\bar{T}_{n+1}^{i}$

2. Solve the fractional momentum equation (Equation (81)), obtain $\tilde{\mathbf{v}}$

3. Solve the continuity equation for the pressure (Equation (82)), obtain $\bar{p}_{n+1}^{i}$

4. Obtain $\bar{\rho}_{n+1}^{i}$ from the ideal gas equation (Equation (4))

5. Solve for end-of-step momentum (Equation (83)) and obtain $\overline{\mathbf{v}}_{n+1}^{i}$

6. Check convergence in terms of density; if converged, go to next time step. Otherwise go to 7.

7. Solve the energy equation implicitly using $\bar{p}_{n+1}^{i}, \bar{\rho}_{n+1}^{i}, \overline{\mathbf{v}}_{n+1}^{i}$

8. Go to 2

\section{EXAMPLES}

The examples chosen show the applicability of the method to the problems involving convection induced by temperature gradients. Attention is paid to the frequency spectra of the solution, reflecting the impact of the presence of the acoustic waves. Detailed comparison with the results of the chosen benchmarks published in literature serves for validating the method. The results shown next correspond to the algorithm, summarized in Table I. The finite element formulation was implemented within the Kratos Multiphysics FE System, a C++ object oriented FE framework developed at the International Center for Numerical Methods in Engineering [16].

\subsection{Natural convection in a square cavity at low Prandtl number}

Natural convection in a square cavity is a classical benchmark example for the solvers for thermally coupled flows. The problem is sketched in Figure 1. The isothermal vertical walls are kept at constant but different temperatures. Upper and lower walls are modeled adiabatic. Homogeneous

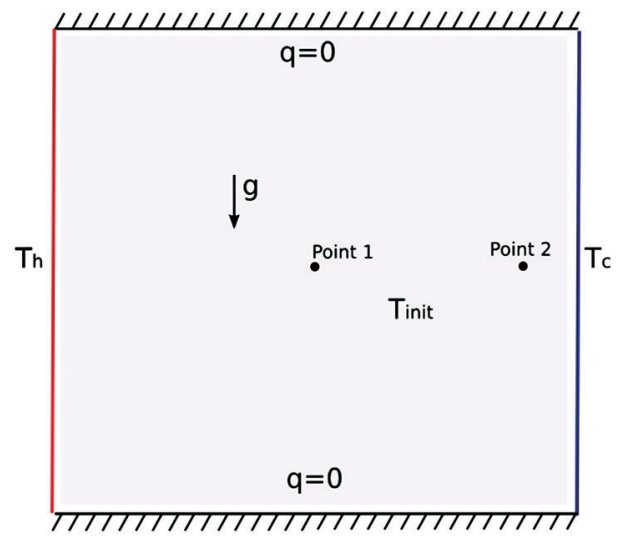

Figure 1. Model of a square heated cavity. 
Dirichlet boundary conditions are prescribed everywhere on the boundary for the velocity. The problem is completely defined by two dimensionless parameters, the Prandtl number $\mathrm{Pr}$ and the Rayleigh number $R a$, defined as

$$
\operatorname{Pr}=\frac{c_{p} v \rho}{k}
$$

and

$$
R a=P r \cdot G r
$$

with $G r$ being the Grashof number

$$
G r=\frac{g L^{3}\left(T_{h}-T_{c}\right)}{v^{2}\left(T_{a v}\right)}
$$

where $c_{p}$ is the specific isobaric heat, $k$ is the conductivity, $T_{h}$ and $T_{c}$ are the temperatures of the hot and cold walls, respectively, $T_{a v}$ is their average, and $L$ is the size of the cavity.

For the validation, numerical results will be presented for $P r=0.01$ and $G r=10 E 7$ according to the benchmark paper of Mohamad and Viskanta [17], where the benchmark is described. Commonly modeled fluids, such as air or water have much higher values of Prandtl numbers, $P r_{\text {water }} \approx 7.0$ and $P r_{\text {air }} \approx 0.7$. For such Prandtl number, the fluid exhibits a steady-state solution. However, it was discovered, that fluids with low Prandtl numbers (these are e.g. liquid semiconductors), constitute an interesting problem: starting from $G r \approx 5 \cdot 10 E 6$, the fluids start exhibiting an oscillatory flow field and a periodic solution. The transient nature of the flow provides a challenging example for testing the solver. Computation of velocity and temperature fields as well as frequency spectra under such flow conditions shall give us a solid base for the validation of the solver developed. As reported in literature, over-diffusive models lead to steady-state instead of periodic solutions. The example allows us also to see the impact that the presence of acoustic waves has upon the solution in a low Mach regime $(M a \approx 0.01)$.

Remark The settings are chosen as follows:

- $T_{h}=305[\mathrm{~K}]$

- $T_{c}=295[\mathrm{~K}]$

- $T_{\text {init }}=295[\mathrm{~K}]$

- $\operatorname{Pr}=0.01$

- $\mathrm{Gr}=10 E 7$

Nondimensionalization was obtained using the following scales: $\Delta T, \frac{L^{2}}{\alpha}, \frac{\alpha}{L}$ for temperature, length, time, and velocity, respectively, where $\alpha=\frac{k}{c_{p} \rho}$ is the thermal diffusivity.

Figure 2 illustrates the temperature and velocity distributions once a periodic behavior is reached. One can see a rotational velocity field. The density distribution is inversely proportional to the temperature, as the pressure distribution is typically hydrostatic.

For the detailed analysis, two sampling points are chosen according to [17]: Point 1 located at the middle of the cavity $(0.5,0.5)$, and Point 2 located at the middle right part of the cavity $(0.9,0.5)$. Next, we present figures displaying the horizontal velocity evolution at these points. The upper graphs correspond to Point 1 and the lower ones to Point 2. Figure 3 illustrates the frequency spectra obtained on three different meshes (all meshes used were uniform and unstructured. Coarse mesh: 2500 elements, standard mesh: 5700 elements, fine mesh: 23000 elements). Comparing the frequency spectra with the results obtained in [17] shown on Figure 4, one can see the dominating frequency of $\approx 25[\mathrm{~Hz}]$ at the cavity center. In our work we obtain one more frequency of $\approx$ 45 [Hz]. Similarly, at Point 2, the two dominating frequencies detected in the numerical experiment in [17] are found in the present work, whereas a third frequency of $\approx 70[\mathrm{~Hz}]$ is also found. Comparison in the time domain and frequency domains show good agreement with results of [17]. The presence of the higher frequencies in the compressible model is expected and reflects the presence of acoustic waves. One can see that as the mesh resolution increases the captured frequency range extends. 


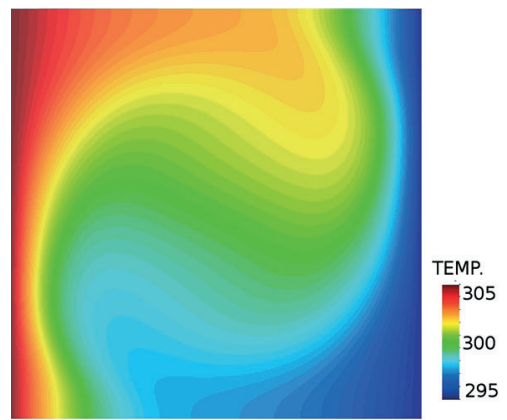

(a) temperature distribution

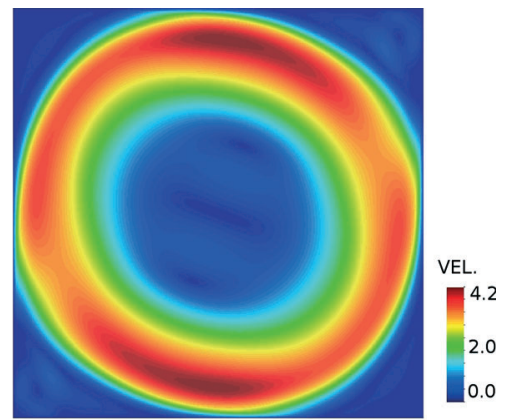

(b) velocity distribution

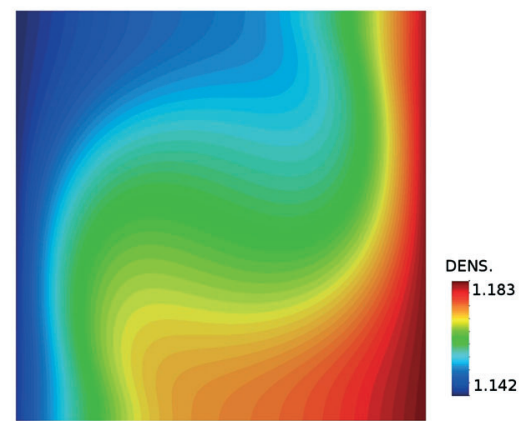

(c) density distribution

Figure 2. Transient behavior in the square heated cavity for $\operatorname{Pr}=0.01, \mathrm{Gr}=10 \mathrm{E} 7$.
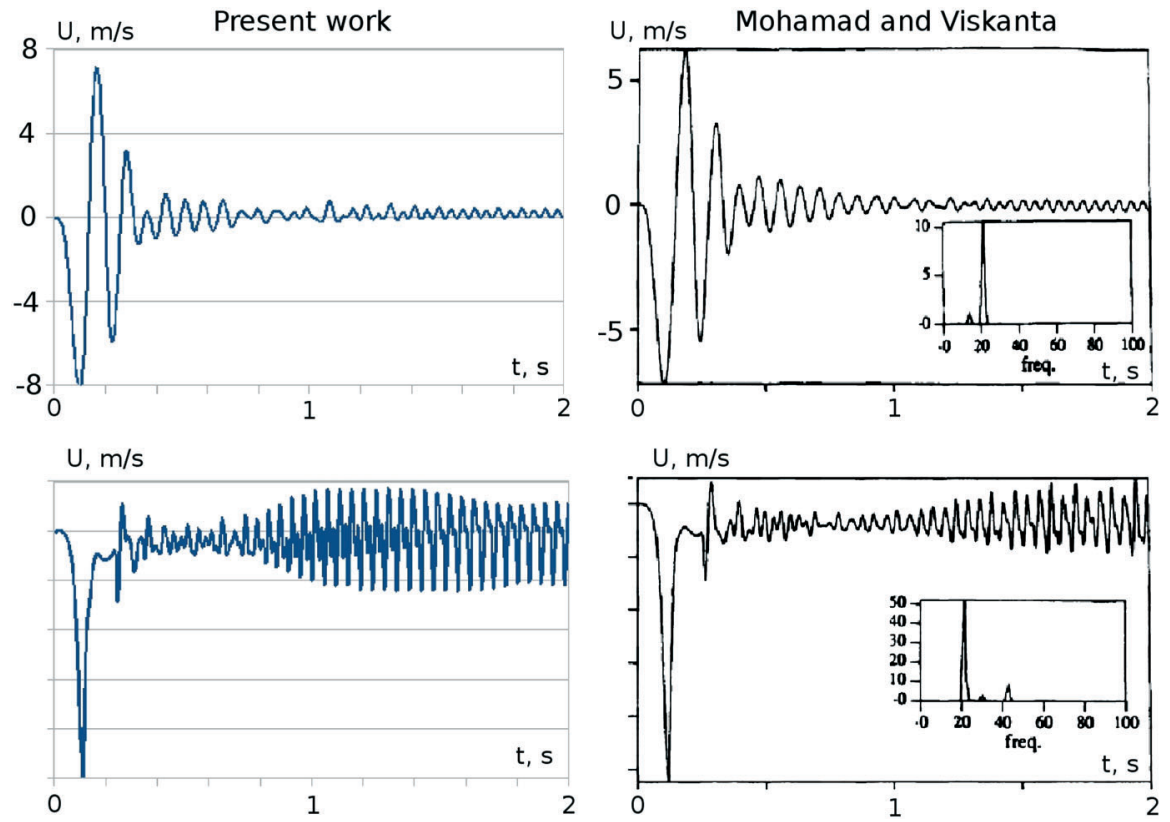

Figure 3. Frequency spectra in the square heated cavity at two sampling points. Results obtained on various meshes.

Remark The average CPU time associated to the solution of one time step for the proposed semi-explicit methodology was compared with the time needed for an implicit solution. For example, for the mesh of 5700 elements and a time step size of $\approx 0.001[\mathrm{~s}]$, the proposed method required $\approx 0.096[\mathrm{~s}]$ per time step, whereas for the equivalent implicit method, the CPU time was of 
Point 1

$(0.5,0.5)$

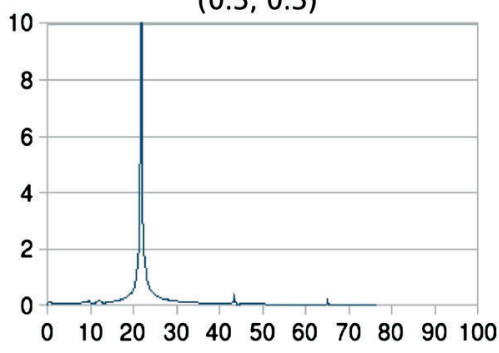

Point 2

$(0.9,0.5)$

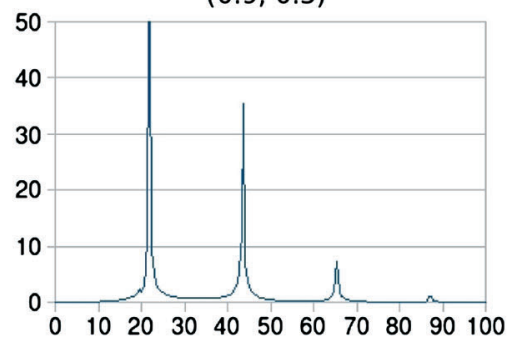

fine mesh
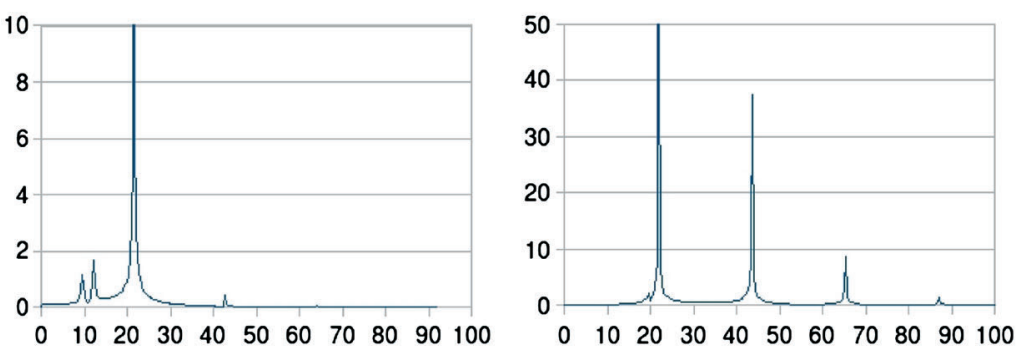

interm. mesh
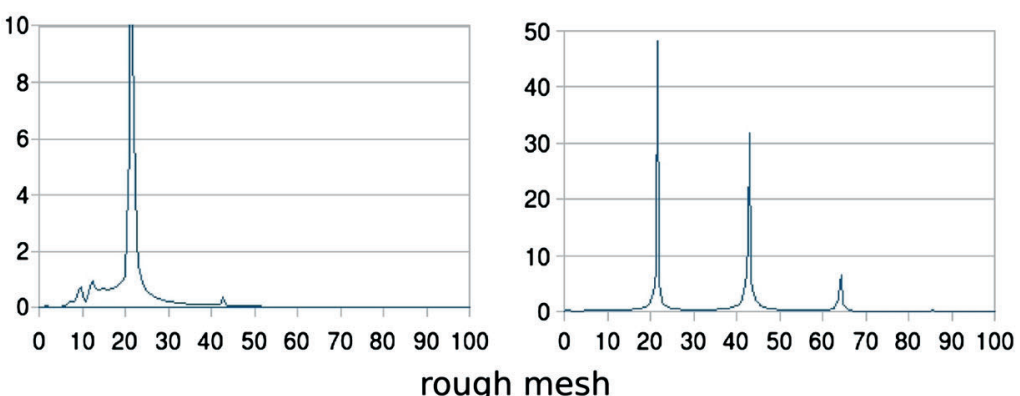

Figure 4. Velocity evolution and frequency spectra in the square heated cavity for $\operatorname{Pr}=0.01, \mathrm{Gr}=10 \mathrm{E} 07$. Comparison with [17].

$\approx 1.42$ [s]. The solutions were obtained with a OpenMP-parallel code using eight processors in both cases. Although this information is clearly implementation-dependent and does not reflect the advantages of the implicit method (like the possibility of using larger time steps), it nevertheless provides some qualitative insight of the computational efficiency of the method.

\section{2. $8: 1$ heated cavity}

Here, the benchmark proposed by Christon et al. in [18] is solved. The example models a differentially heated rectangular cavity. The ratio between the vertical/horizontal edge length is $8: 1$. The spatial structure of the flow is made of vertical and horizontal boundary layers and corner structures, which depend very sensitively on the aspect ratio, Prandtl number, and thermal boundary conditions. All these features lead to a very complex time behavior resulting from several instability mechanisms, traveling waves in the vertical boundary layers, and thermal instabilities along the horizontal walls [19].

The geometry is shown in Figure 5, and the benchmark parameters are summarized next:

- $\mathrm{L}=1 \mathrm{~m}$

- $\mathrm{H}=8 \mathrm{~m}$

- $T_{h}=285.15[\mathrm{~K}]$ 


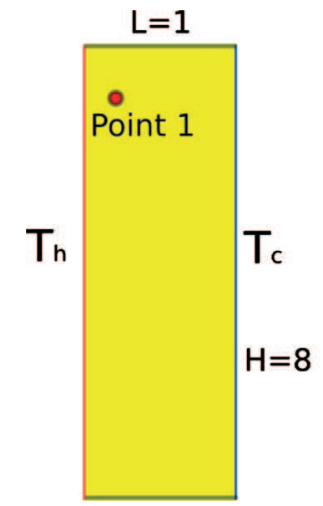

(a) model and location of the reference point

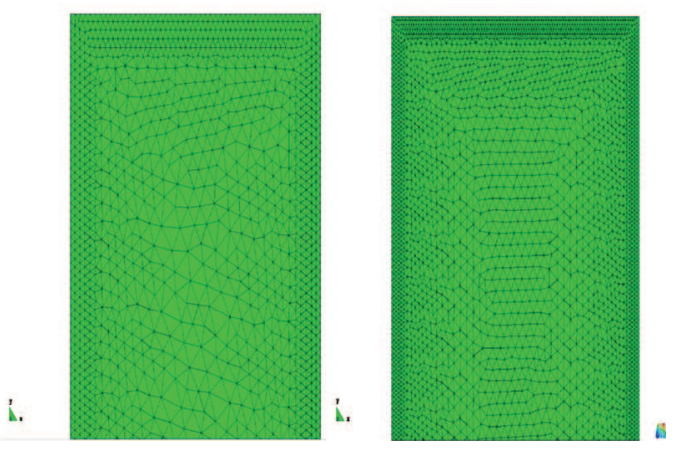

(b) meshes used

Figure 5. Differentially heated cavity with an aspect ratio of 8:1

- $T_{c}=275.15[\mathrm{~K}]$

- $R a=3.4 E 05$

- $\operatorname{Pr}=0.71$

- Point of analysis: $(x, y)=(0.181,7.37)$

- top and bottom walls are adiabatic.

The model is shown schematically in Figure 5. Nondimensionalization was obtained using the following scales: $\Delta T, L, \sqrt{g \beta L \Delta T}, L / \sqrt{g \beta L \Delta T}$ for temperature, length, time, and velocity, respectively, where $\beta=1 / T_{a v}$ is the thermal expansion coefficient. All the data will be presented in terms of nondimensionalized quantities. Tests were carried out using two meshes, one containing 17000 (referred as 'coarse') triangles with size varying from 0.02 (at the walls) to 0.08 (in the interiors) and the other one containing 45000 (referred as 'fine') with sizes varying from 0.01 (at the walls) to 0.03 (in the interiors). Both meshes are shown in Figure 5.

In establishing this benchmark, Christon et al. [18] proposes $R a=3.4 E 05$ as it constitutes a slightly over-critical Rayleigh number. All the investigations carried out [19-22] announce critical Rayleigh number to be above 3.0E05. To ensure that in the under-critical regime the flow converges to the steady behavior, we performed a series of tests with $R a=2.8 E 05$. The solution converged to a steady-state independent of the mesh used conforming the expected result (this can be seen in Figure 6, where the temperature evolution at 'Point 1' defined by Christon [19] as $(x, y)=(0.1810,7.37)$ is shown $)$.

Next, we present the results obtained on two meshes with the compressible solver developed in this work in the critical regime; that is, when instead of a steady-state, a periodic solution is reached.

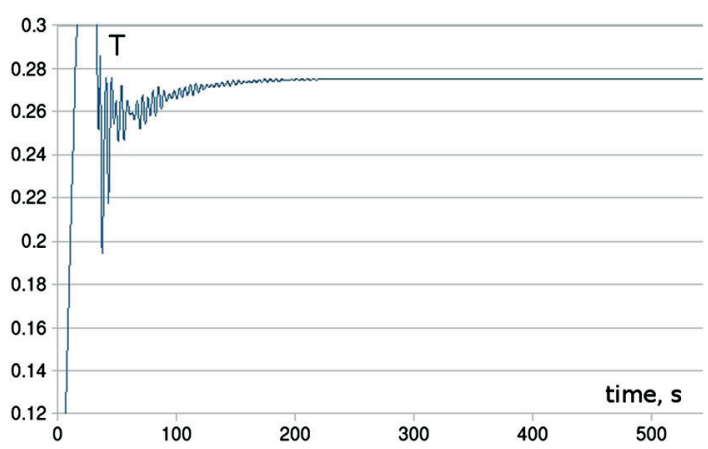

Figure 6. Temperature (nondimensionalized) evolution for $\mathrm{Ra}=2.8 \mathrm{E} 05$ (under-critical regime) in the 8:1 cavity. 
The comparison of the compressible solver results on coarse and fine meshes is shown in Figure 7(a). One can see that the oscillation amplitude does not vary considerably between both meshes (see the superimposed graphs). The average temperature value is found to be $\Theta=0.265888$, and the average amplitude $a=0.007377$. The period of oscillations is found to be 3.51. The comparison with the results of Christon [18] are summarized in Table III.

It was observed in [19] that explicit algorithms on coarse meshes were unable to capture the oscillatory solution found at $R a=3.4 \cdot 10 E 05$ and $\operatorname{Pr}=0.71$. The cause was attributed to the mass lumping used in the explicit schemes. In the present work in spite of the mass lumping, the periodic behavior was captured already on the rough mesh using the present methodology, this can be seen on Figure 8. A comparison with the explicit Boussinesq assumption-based solver is also shown on Figure 8. The solver based on the Boussinesq assumption led to under-estimation of the oscillation amplitude on the fine mesh, whereas not capturing the oscillating behavior on the rough mesh.

The stream-lines pattern obtained by solving the problem with the compressible solver is shown in Figure 7(b). The results match very well those reported by Salinger et al. in [22], namely the stream-line pattern corresponding to the flow after the first Hopf bifurcation. However, in [22], the critical Rayleigh number was found to be $R a_{c r}=3.61 \cdot E 05$, which is larger than the ones reported by Xin and Le Quere [21] and Christon [18], that found $R a_{c r}=3.1 \cdot E 05$. The present results show that $R a_{c r}=3.4 \cdot E 05$ correspond to the over-critical regime.

\section{SUMMARY AND CONCLUSIONS}

A finite element formulation and an algorithm for the solution of compressible subsonic flows was proposed. The formulation was based on the complete set of compressible Navier-Stokes equations.

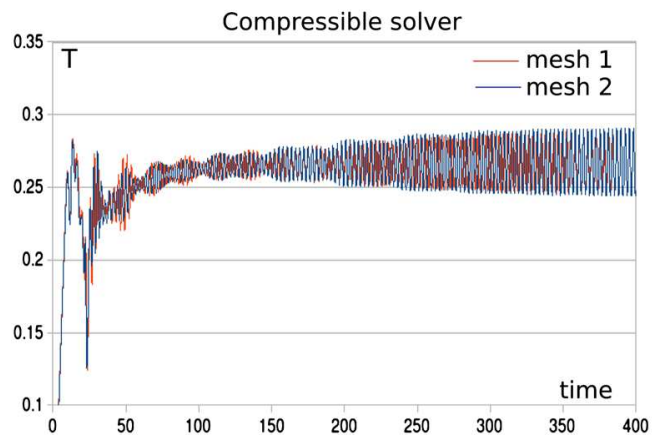

(a) Results obtained on rough and fine meshes super imposed

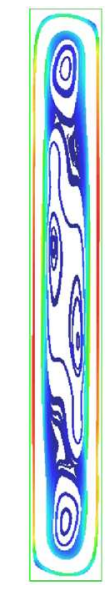

(b) stream lines pattern

Figure 7. Temperature (nondimensionalized) evolution for Ra $=3.4$ E05 (supercritical regime) in the 8:1 cavity.

Table III. Comparison of the compressible solver results with the ones obtained in [19] for the 8:1 cavity.

\begin{tabular}{lcc}
\hline Parameter & Comp. solver & Christon(ref.mesh) \\
\hline Period avg & 3.51 & 3.52 \\
$u_{x}$ avg & 0.0608 & 0.06112 \\
$u_{x}$ ampl & 0.0521 & 0.05078 \\
$u_{y}$ avg & 0.4473 & 0.4638 \\
$u_{y}$ ampl & 0.07724 & 0.0726 \\
T avg & 0.26588 & 0.2663 \\
T ampl & 0.0421 & 0.03975 \\
\hline
\end{tabular}



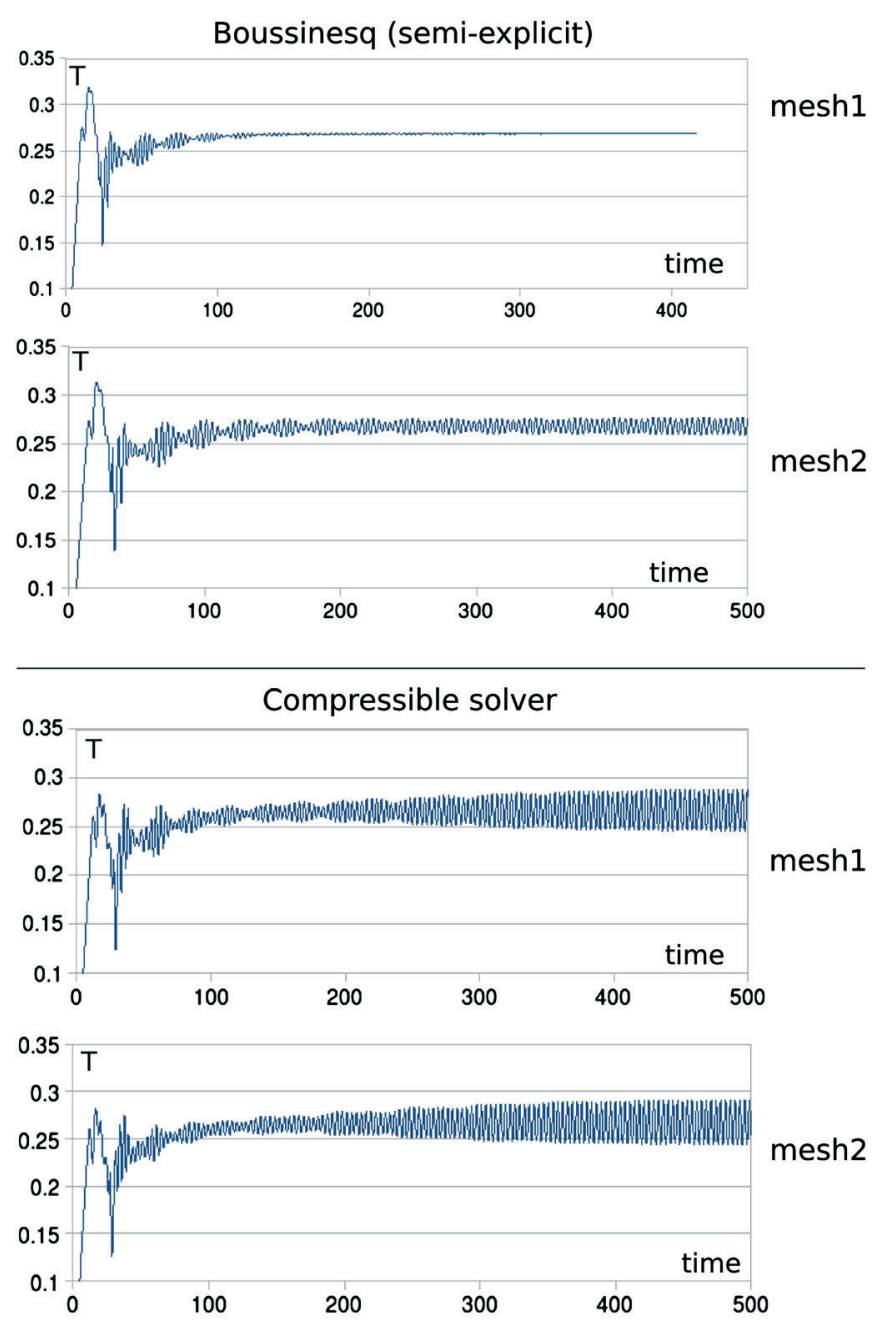

Figure 8. Temperature (nondimensionalized) evolution for $\mathrm{Ra}=3.4 \mathrm{E} 05$ in the 8:1 cavity.

The absence of shock waves in the subsonic regime permitted to write the governing equations in the nonconservation form. We showed that in the case of explicit treatment of the energy equation, this becomes uncoupled from the rest of the system naturally (without the need for introducing any approximation). For solving the (uncoupled) momentum/continuity system, we proposed a semiexplicit method, where the momentum equation was integrated explicitly (except for the pressure term) and the continuity-implicitly using a dissipative time integrator. We showed that under the assumption of linear variation of temperature within a time step, one may omit resolving the continuity equation at the intermediate steps of the Runge-Kutta scheme, thus reducing the computational effort associated to the implicit step. The features of the method can be summarized as:

- efficiency close to that of a fully explicit approach

- implicit treatment of pressure leading to the invariance of the time step estimation of the acoustic waves

- the frequency spectrum contains the acoustic contributions that can be correctly resolved by FEM

- spurious high frequencies are damped by a dissipative time integrator

We also addressed a possibility of an iterative approach, where the solution of the energy equation based on the known values of velocity, pressure, density is considered to be a guess for the momentum/equation system, that is updated until convergence in temperature is achieved. In either case, 
the fractional step split was applied to the momentum/continuity system. The introduced methodology not only permits splitting the monolithic system into a set of smaller ones, but also a modular implementation similar to that of the incompressible case.

\section{ACKNOWLEDGEMENTS}

The authors would like to thank the International Center for Numerical Methods in Engineering (CIMNE) for kindly supporting this work. The work is also supported under the auspices of the 'eDams' project of the Spanish Ministry of Science and Education (BIA2010-21350-C03-00) and the SAFECON project of the European Research Council (ERC). Special thanks are given to Dr. J. Marti for providing us the details regarding the CPU times associated with the proposed method.

\section{REFERENCES}

1. Nikulin DA. Applicability of the Boussinesq approximation for the solution of problems of unsteady natural concentration convection. Fluid Dynamics 1982; 17(5):779-781.

2. Gartling DK, Hickox CE. A numerical study of the applicability of the Boussinesq approximation for a fluid-saturated porous medium. International Journal for Numerical Methods in Fluids 2005; 5(11):995-1013.

3. Gray DD, Giorgini A. The validity of the Boussinesq approximation for liquids and gases. International Journal of Heat and Mass Transfer 1976; 15:545-551.

4. Principe J. Subgrid scale stabilized finite elements for low speed flows. Ph.D. Thesis, Technical University of Catalonia, 2008.

5. Rehm R, Baum H. The equations of motion for thermally driven bouyant flows. Journal of Research of the National Bureau of Standards 1975; 83:297-308.

6. Müller B. Low Mach number asymptotics of the navier-stokes equations. Journal of Engineering Mathematics 1998; 34:97-109.

7. Principe J, Codina R. On the the low Mach number and the Boussinesq approximations for low speed flows. International Journal for Numerical Methods in Fluids, submitted.

8. Principe J, Codina R. A stabilized finite element approximation of low speed thermally coupled flows. International Journal for Numerical Methods for Heat and Fluid flow 2008; 18:835-867.

9. Donea J, Huerta A. Finite Element Methods for Flow Problems. J. Wiley: Chichester, England, 2003.

10. Zienkiewizc OC, Codina R. A general algorithm for compressible and incompressible flow part i. the split, characteristic-based scheme. International Journal for Numerical Methods in Fluids 1995; 20:869-885.

11. Hauke G, Hughes T. A unified approach to compressible and incompressible flows. Computer Methods in Applied Mechanical Engineering 1994; 113:389-395.

12. Codina R, Vazquez M, Zienkiewizc OC. A general algorithm for compressible and incompressible flows. International Journal for Numerical Methods in Fluids 1998; 27:13-32.

13. Brezzi F, Bathe K-J. A discourse on the stability of the mixed finite element formulations. Journal of Computer Methods in Applied Mechanics 1990; 22:27-57.

14. Codina R. Stabilized finite element approximation of transient incompressible flows using orthogonal subscales. Computer Methods in Applied Mechanics and Engineering 2002; 191:4295-4321.

15. Oñate E. A stabilized finite element method for incompressible viscous flows. Computer Methods in Applied Mechanics and Engineering 2000; 182(1-2):355-370.

16. Dadvand P, Rossi R, Oñate E. An object-oriented environment for developing finite element codes for multidisciplinary applications. Archives of Computational Methods in Engineering; 17(3):253-297.

17. Mohamad AA, Viskanta R. Transient natural convection of low-prandtl-number fluids in a differentially heated cavity. International Journal for Numerical Methods in Fluids 1991; 13:61-81.

18. Christon MA, Gresho PM, Sutton SB. Computational predictability of time-dependent natural convection flows in enclosures (including benchmark solution). International Journal for Numerical Methods in Fluids 2002; 40:953-980.

19. Christon MA. Ls-dyna and the 8:1 differentially heated cavity. International Journal for Numerical Methods in Fluids 2002; 40:1133-1144.

20. Gresho PM, Sutton SB. Application of the fidap code to the 8:1 thermal cavity problem. International Journal for Numerical Methods in Fluids 2002; 40(8):1083-1092.

21. Xin S, Le Quere P. An extended Chebyshev pseudo-spectral benchmark for the 8:1 differentially heated cavity. International Journal for Numerical Methods in Fluids 2002; 40(8):981-998.

22. Salinger AG, Lehoucq RB, Pawlowski RP, Shadid JN. Understanding the $8: 1$ cavity via scalable stability analysis algorithms. First MIT Conference on Computational Fluid and Solid Mechanics, 2001. Conference proceedings. 